\title{
SIPPI: A Matlab toolbox for sampling the solution to inverse problems with complex
} prior information

Part 1-Methodology

Hansen, Thomas Mejer; Cordua, Knud Skou; Caroline Looms, Majken; Mosegaard, Klaus

Published in:

Computers \& Geosciences

Link to article, DOI:

10.1016/j.cageo.2012.09.004

Publication date:

2013

Link back to DTU Orbit

Citation (APA):

Hansen, T. M., Cordua, K. S., Caroline Looms, M., \& Mosegaard, K. (2013). SIPPI: A Matlab toolbox for sampling the solution to inverse problems with complex prior information: Part 1-Methodology. Computers \& Geosciences, 52, 470-480. https://doi.org/10.1016/j.cageo.2012.09.004

\section{General rights}

Copyright and moral rights for the publications made accessible in the public portal are retained by the authors and/or other copyright owners and it is a condition of accessing publications that users recognise and abide by the legal requirements associated with these rights.

- Users may download and print one copy of any publication from the public portal for the purpose of private study or research.

- You may not further distribute the material or use it for any profit-making activity or commercial gain

- You may freely distribute the URL identifying the publication in the public portal 


\section{Author's Accepted Manuscript}

SIPPI: A Matlab toolbox for sampling the solution to inverse problems with complex prior information: Part 1 - Methodology

Thomas Mejer Hansen, Knud Skou Cordua, Majken Caroline Looms, Klaus Mosegaard

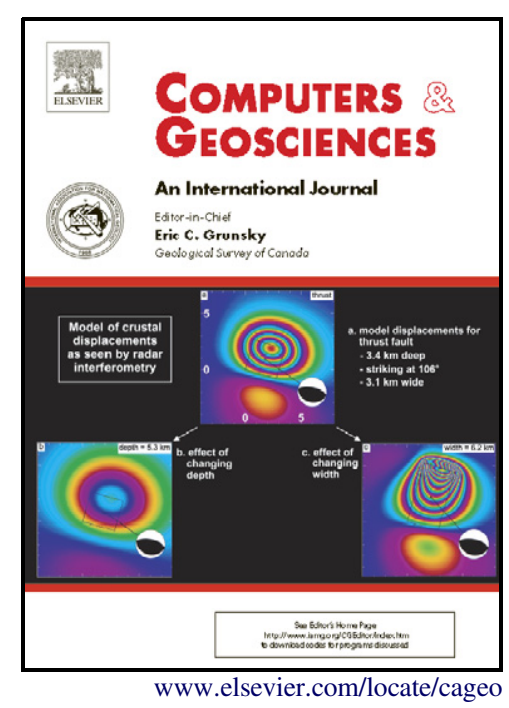

PII:

S0098-3004(12)00313-5

DOI: http://dx.doi.org/10.1016/j.cageo.2012.09.004

Reference: CAGEO3018

To appear in: Computers \& Geosciences

Received date: 22 June 2012

Revised date: 7 September 2012

Accepted date: 10 September 2012

Cite this article as: Thomas Mejer Hansen, Knud Skou Cordua, Majken Caroline Looms and Klaus Mosegaard, SIPPI: A Matlab toolbox for sampling the solution to inverse problems with complex prior information: Part 1 - Methodology, Computers \& Geosciences, http://dx.doi.org/10.1016/j.cageo.2012.09.004

This is a PDF file of an unedited manuscript that has been accepted for publication. As a service to our customers we are providing this early version of the manuscript. The manuscript will undergo copyediting, typesetting, and review of the resulting galley proof before it is published in its final citable form. Please note that during the production process errors may be discovered which could affect the content, and all legal disclaimers that apply to the journal pertain. 


\title{
SIPPI : A Matlab toolbox for sampling the solution to inverse problems with complex prior information: Part 1 - Methodology
}

\author{
Thomas Mejer Hansen ${ }^{\mathrm{a}, *}$, Knud Skou Cordua ${ }^{\mathrm{a}}$, Majken Caroline Looms ${ }^{\mathrm{b}}$, \\ Klaus Mosegaard ${ }^{\mathrm{a}}$ \\ ${ }^{a}$ Technical University of Denmark, Center for Energy Resources Engineering, DTU \\ Informatics, Asmussens Alle, Building 305, DK-2800 Lyngby, Denmark \\ ${ }^{b}$ University of Copenhagen, Department of Geography og Geology, Øster Voldgade 10, \\ DK-1350 København K, Denmark
}

\begin{abstract}
From a probabilistic point-of-view, the solution to an inverse problem can be seen as a combination of independent states of information quantified by probability density functions. Typically, these states of information are provided by a set of observed data and some a priori information on the solution. The combined states of information (i.e. the solution to the inverse problem) is a probability density function typically referred to as the a posteriori probability density function. We present a generic toolbox for Matlab and Gnu Octave called SIPPI that implements a number of methods for solving such probabilistically formulated inverse problems by sampling the a posteriori probability density function. In order to describe the a priori probability density function, we consider both simple Gaussian models and more complex (and realistic) a priori models based on higher order statistics. These a priori models can be used with both linear and non-linear inverse problems. For linear inverse Gaussian problems we make use of least-squares and kriging-based methods to describe the a posteriori probability density function directly. For general non-linear (i.e. non-Gaussian) inverse problems we make use of the extended Metropolis algorithm to sample the a posteriori
\end{abstract}

*Corresponding author. Tel.:+45 45253086, Fax.: +45 45882673

Email addresses: tmeha@imm.dtu.dk (Thomas Mejer Hansen), kcor@imm.dtu.dk (Knud Skou Cordua), mcl@geol.ku.dk (Majken Caroline Looms), kmos@imm.dtu.dk (Klaus Mosegaard) 
probability density function. Together with the extended Metropolis algorithm we use sequential Gibbs sampling that allow computationally efficient sampling of complex a priori models. The toolbox can be applied to any inverse problem as long as a way of solving the forward problem is provided. Here we demonstrate the methods and algorithms available in SIPPI. An application of SIPPI, to a tomographic cross borehole inverse problems, is presented in a second part of this paper.

Keywords: inversion, nonlinear, sampling, a priori, a posteriori

\section{Introduction}

Inverse problems are abundant in almost any type of scientific research

3 field. An inverse problem occurs when a set of unknown parameters, that

4 describe a physical system, pixel values of an image or some mathematical

5 expression, have to be inferred based on indirect observations of these pa-

6 rameters. Examples of inverse problems are image debluring, tomographic

7 reconstruction, solutions to certain differential equations, or reconstructing

8 the earth's interior based on surface observations. There are several ways to

9 solve an inverse problem. In a probabilistic formulation the inverse problem

10 can be seen as a way of combining information: Given knowledge about the

system (differential equation, physical law, or blurring mechanisms), and a set of observations (signal intensities, pixel values, gravity field), and some prior expectations about the parameters, the goal is to quantify how probable a number of possible scenarios are of explaining the observations and

locate all solutions to the problem and assign a probability to each scenario given the information at hand. 
In this paper we present a Matlab ${ }^{1}$ toolbox (SIPPI), compatible with Gnu Octave $^{2}$, that can be used to solve inverse problems in a probabilistic formulation. In this formulation the solution to the inverse problem is a probability density function (pdf) referred to as the a posteriori pdf, that describe all information available about a system. While the toolbox is generally applicable to inverse problems, it has been designed specifically for geophysical inverse problems, where the model parameters typically describe a 1D-3D space, such as for example the subsurface of the earth.

Initially we lay out the theory of probabilistically formulated inverse problems. Then we show how so-called a priori information about the model parameters, and uncertainty of data observations can be specified. Finally we show how realizations of the a posteriori pdf can be generated using least squares based methods, and sampling techniques such as rejection sampling and Metropolis sampling.

In a second part of this manuscript we demonstrate the application of SIPPI to a cross borehole traveltime tomographic inverse problem, Hansen et al. (this issue).

\section{Probabilistic Inverse Problem Theory}

Consider some data, $\mathbf{d}$, which are indirect measurements of some model parameters, $\mathbf{m}$, describing a system, such as for example the subsurface of the Earth. Let $\mathbf{d}$ and $\mathbf{m}$ be related through the function $g$ :

$$
\mathbf{d}=g(\mathbf{m})
$$

\footnotetext{
${ }^{1} \mathrm{http}: / /$ mathworks.com/

${ }^{2}$ http://www.gnu.org/software/octave
} 
Eq. 1, referred to as the forward problem, can be solved with various degrees of accuracy for a number of physical problems.

Inversion of geophysical data amounts to infer information about the model parameters, $\mathbf{m}$, given some data, $\mathbf{d}$, the forward relation between model parameters and data, $g$, and a priori existing knowledge about the model parameters. Such an inverse problem can be solved in a variety of ways. In this paper we will deal with the general probabilistic formulation of inverse problems. Note that many types of deterministic inversion methods can be formulated as special cases of the probabilistic inverse theory as we consider here.

Tarantola and Valette (1982b) formulate a probabilistic approach for solving inverse problems where all available states of information is described by pdfs. The solution to the inverse problem is the pdf that combines known states of information. In a typical inverse problem the states of information can be described by the a priori pdf and the likelihood function. The a priori pdf, $\rho_{\mathrm{M}}(\mathbf{m})$, describes prior knowledge about the model parameters. The likelihood function, $L(\mathbf{m})$, is a probabilistic measure of how well a given model $\mathbf{m}$ explains observed data.

The general solution to such a probabilistically formulated inverse problem is the a posteriori pdf, which is proportional to the product of the a priori pdf and the likelihood function:

$$
\sigma_{\mathrm{M}}(\mathbf{m})=k \rho_{\mathrm{M}}(\mathbf{m}) L(\mathbf{m})
$$

60 where the $k$ is a normalization constant and the likelihood is given by

$$
L(\mathbf{m})=\int_{\mathcal{D}} d \mathbf{d} \frac{\rho_{\mathrm{D}}(g(\mathbf{m})) \theta(\mathbf{d} \mid \mathbf{m})}{\mu_{\mathrm{D}}(\mathbf{d})}
$$


$\rho_{\mathrm{D}}(\mathbf{d})$ describes measurement uncertainties, typically related to uncertainties in the instrument that records the data. $\theta(\mathbf{d} \mid \mathbf{m})$ describes the modelization error, i.e. the error caused by using an imperfect forward model $g$ or an imperfect parameterization. $\mu_{\mathrm{D}}(\mathbf{d})$ describes the homogeneous state of information that ensures that the parameterization is invariant to changes in the coordinate system. For the reminder of the text we shall assume that $\mu_{\mathrm{D}}(\mathbf{d})$ can be approximated by a constant. For more details on the homogeneous pdf see e.g. Mosegaard and Tarantola (2002).

The a posteriori pdf describes the distribution of models consistent with the combined states of information given by the a priori model and the data.

The probabilistic formulation of inverse problems allows utilization of the movie strategy advocated by Tarantola (2005), who suggest to visualize and compare a sample from the a priori pdf and the a posteriori pdf, respectively, as movies. The 'prior movie' will make it apparent what prior choices have been made. The difference between the prior and the posterior movie will emphasize the effect of using data.

\subsection{The linear inverse Gaussian problem}

Consider a linear forward problem, where the data $\mathbf{d}$ is linearly related to the model parameters $\mathbf{m}$ using the linear operator $\mathbf{G}$, such that $\mathbf{d}=\mathbf{G m}$. Let $\mathcal{N}(\mathbf{a}, \mathbf{A})$ refer to a Gaussian distribution with mean a and covariance $\mathbf{A}$. If in addition both the a priori model $\mathcal{N}\left(\mathbf{m}_{0}, \mathbf{C}_{\mathrm{M}}\right)$, the noise model $\mathcal{N}\left(0, \mathbf{C}_{d}\right)$ and the modelization error $\mathcal{N}\left(0, \mathbf{C}_{T}\right)$ can be described by a Gaussian pdf, then the a posteriori pdf (Eq. 2) can be described analytically by a Gaussian pdf, $\mathcal{N}\left(\widetilde{\mathbf{m}}, \widetilde{\mathbf{C}}_{\mathrm{M}}\right)$ (Tarantola and Valette, 1982a):

$$
\widetilde{\mathbf{m}}=\mathbf{m}_{0}+\mathbf{C}_{\mathrm{M}} \mathbf{G}^{t}\left(\mathbf{G C}_{\mathrm{M}} \mathbf{G}^{\prime}+\mathbf{C}_{\mathrm{D}}\right)^{-1}\left(\mathbf{d}_{0}-\mathbf{G} \mathbf{m}_{0}\right)
$$




$$
\widetilde{\mathbf{C}}_{\mathrm{M}}=\mathbf{C}_{\mathrm{M}}-\mathbf{C}_{\mathrm{M}} \mathbf{G}^{t}\left(\mathrm{GC}_{\mathrm{M}} \mathrm{G}^{\prime}+\mathrm{C}_{\mathrm{D}}\right)^{-1} \mathbf{G C}_{\mathrm{M}}
$$

Note that Gaussian measurement errors and modelization errors combine through addition of the covariance operators, such that the combined covariance model is given by $\mathbf{C}_{\mathrm{D}}=\mathbf{C}_{d}+\mathbf{C}_{T}$. This allows accounting of Gaussian modelization errors directly as given in Eqs. 4-5, Tarantola (2005).

If $\widetilde{\mathbf{m}}$ and $\widetilde{\mathbf{C}}_{\mathrm{M}}$ are available from Eqs. 4-5 then samples from the a posteriori pdf can be generated using e.g. Cholesky decomposition of the a posteriori covariance model, Eq. 5 in Le Ravalec et al. (2000).

Sampling the a posteriori pdf of a linear inverse Gaussian problem can also be performed using sequential Gaussian simulation without the need for explicitly computing $\widetilde{\mathbf{m}}$ and $\widetilde{\mathbf{C}}_{\mathrm{M}}$, Hansen et al. (2006). Hansen and Mosegaard (2008) extend this approach to work with direct sequential simulation. This allows a non-Gaussian a priori distribution of model parameters.

An alternative approach is to use kriging through error simulation, Journel and Huijbregts (1978, p. 495), in a co-kriging formulation as proposed by Gloaguen et al. $(2004,2005)$. This approach may be faster than the methods based on sequential simulation, but is only valid for strictly Gaussian a priori models.

The above mentioned methods rely on the fact that in a linear formulation, data can be seen as weighed averages of the model parameters. While not specifically making the link to inverse problems, such ideas has also been explored by Journel (1999) and Gómez-Hernández et al. (2005).

\subsection{The non-linear Inverse problem}

The linear and Gaussian assumptions considered above are convenient as they lead to computationally efficient algorithms. However, in reality 
the inverse problem is typically non-linear and the Gaussian assumption not valid. This may lead to severe artifacts in the inversion if the least-squares based approaches, as described above, are used. Instead one can use sampling techniques to sample the a posteriori pdf.

Rejection sampling. Perhaps the simplest method to sample the a posteriori pdf is the rejection sampler, that can be implemented as follows

1. Propose a model candidate from the a priori pdf, $\mathbf{m}_{\text {pro }}$.

2. Compute $L\left(\mathbf{m}_{\text {pro }}\right)$

3. Accept the proposed model as a realization of the a posteriori pdf with probability

$$
P_{\text {acc }}=L\left(\mathbf{m}_{\text {pro }}\right) / L_{\max }
$$

where $L_{\max }$ is the maximum value the likelihood function can obtain. Typically the value of $L_{\max }$ is not known and must be set to 1 . The only requirements for using the method is that one must be able to generate independent realizations of the a priori pdf and compute the corresponding likelihood. The collection of models accepted by the rejection sampling algorithm will be a sample of the a posteriori pdf. The main problem with the rejection sampler is that it is computationally very inefficient for anything but very low dimensional problems.

The extended Metropolis sampler. Mosegaard and Tarantola (1995) propose an extended version of the Metropolis algorithm (Metropolis et al. (1953); Hastings (1970)) that allows sampling the a posteriori pdf of an inverse problem with, in principle, arbitrary complex a priori information as given by Eq. 2. Using the classical Metropolis algorithm one must be able to 
evaluate the a posteriori probability $\sigma_{\mathrm{M}}(\mathbf{m})$ and, hence, typically also the a priori probability, in order to evaluate Eq. 2.

The extended Metropolis algorithm differ from the classical Metropolis algorithm in that one does not need to evaluate the a posteriori probability $\sigma_{\mathrm{M}}(\mathbf{m})$, nor the a priori probability $\rho_{\mathrm{M}}(\mathbf{m})$ of a given model $\mathbf{m}$. If only an algorithm is present that can sample the a priori pdf and a method exist for evaluating the likelihood, $\rho_{\mathrm{D}}(g(\mathbf{m}))$, then the extended Metropolis algorithm will sample the a posteriori pdf.

The extended Metropolis algorithm is a Markov Chain Monte Carlo method and can be implemented as a random walk in the space of a priori acceptable models as follows. If initially a realization of the a priori pdf is generated as $\mathbf{m}_{\text {cur }}$, and the associated likelihood $L\left(\mathbf{m}_{\text {cur }}\right)$ is evaluated using Eq. 3, then the following algorithm will sample the a posteriori pdf

1. In the vicinity of $\mathbf{m}_{\text {cur }}$, propose a new model candidate, $\mathbf{m}_{\text {pro }}$, consistent with the a priori model.

2. Compute $L\left(\mathbf{m}_{\text {pro }}\right)$

3. Accept the proposed model with probability $P_{a c c}=\min \left(\left[1, L\left(\mathbf{m}_{\text {pro }}\right) / L\left(\mathbf{m}_{\text {cur }}\right)\right]\right)$

4. If the proposed model is accepted, then the transition from $\mathbf{m}_{c u r}$ to $\mathbf{m}_{\text {pro }}$ is accepted, and the proposed model becomes the current model, $\mathbf{m}_{\text {cur }}=\mathbf{m}_{\text {pro }}$. Otherwise the random walker stays a location $\mathbf{m}_{\text {cur }}$ and $\mathbf{m}_{\text {cur }}$ counts again.

There are only two requirements for running the extended Metropolis algorithm: 1) One must be able to evaluate the likelihood function, Eq. 3. This is most often trivial, even if it may be computationally demanding, as it requires one to solve the forward problem and evaluate the correspond- 
ing data fit given the noise model. 2) One must be able to sample the a priori pdf such that aperiodicity and irreducibility is ensured, Mosegaard and Sambridge (2002). In addition, it is preferable to be able to control the exploratory nature (often referred to as the step length) of the sampling algorithm, i.e. step 1 in the above algorithm, which is closely linked to the computational efficiency. See Mosegaard and Tarantola (1995) for details on the extended Metropolis algorithm.

The sequential Gibbs sampling algorithm provides such a general way to sample complex a priori models, with arbitrary step length ensuring aperiodicity and irreducibility, Hansen et al. (2012). Sequential Gibbs sampling can be used with any pdf that can be sampled using sequential simulation, which is the case for most of the statistical models developed in the geostatistical community over the last decades. The resampling strategy inherent in the sequential Gibbs sampler was initially proposed by Hansen et al. (2008), and subsequently Irving and Singha (2010) and Mariethoz et al. (2010) proposed similar methods. Hansen et al. (2012) demonstrate how the method is similar to an application of the Gibbs sampler and show that the method leads to a way of sampling the a priori pdf where aperiodicity and irreducibility is ensured.

\section{SIPPI}

SIPPI is a Matlab toolbox (SIPPI), compatible with Gnu Octave, that can be used to solve inverse problems in the formulation given by Eqs. 2-3 by allowing Sampling the solution to Inverse Problems with complex A Priori Information. 
In order to solve a probabilistic framed inverse problem as presented previously, one needs (at least) three ingredients: 1) a choice of an a priori model, 2) a choice of how to solve the forward problem, and 3) a choice of a noise model model that describes the uncertainty of the observed data and the modelization error. Once these choices have been made one can solve the inverse problem using any of the applicable inversion methods.

SIPPI provides a generic approach to defining the a priori model and the noise model in form of the two data structures prior and data.

\subsection{The a priori model}

All information about the a priori model is defined in the Matlab structure called prior, which can specify any number of a priori type of models. For example an a priori choice of a 2D Gaussian velocity field can be specified in prior $\{1\}$ and a $1 \mathrm{D}$ parameter describing a bias correction can be specified in prior $\{2\}$. Once the prior has been defined, a realization of the corresponding a priori pdf can be generated by calling

m=sippi_prior (prior);

$\mathrm{m}$ is a Matlab structure of the same size as prior. If 3 types of a priori models have been defined in prior $\{1\}, \operatorname{prior}\{2\}$, and $\operatorname{prior}\{3\}$ then the corresponding realizations will be stored in $\mathrm{m}\{1\}, \mathrm{m}\{2\}$, and $\mathrm{m}\{3\}$. Considering the example above, $\mathrm{m}\{1\}$ will hold a realization of a $2 \mathrm{D}$ a priori model, while $\mathrm{m}\{2\}$ will hold a realization of a $1 \mathrm{D}$ a priori model. For the remainder of the text the index im will point to a specific number of a priori model, $\operatorname{prior}\{i m\}$. 
A number of different types of a priori models can be selected using a type field to the prior data structure. The following 4 types of a priori models are available as part of SIPPI:

im=1;

prior $\{$ im $\}$.type='GAUSSIAN';

prior $\{$ im $\}$.type='FFTMA';

prior $\{$ im $\}$. type=' VISIM';

prior $\{$ im $\}$.type='SNESIM' ;

Generalized Gaussian. prior $\{$ im $\}$.type=GAUSSIAN' defines a 1D generalized Gaussian distribution;

$$
f_{g g}\left(m_{0}, \sigma, p\right)=\frac{p^{1-1 / p}}{2 \sigma \Gamma(1 / p)} \exp \left(-\frac{1}{p} \frac{\left|m-m_{0}\right|^{p}}{\sigma^{p}}\right)
$$

where $p$ is the norm, $\sigma$ the variance. $f_{g g}$ is symmetric around $m_{0}$, the a priori mean value. In the limit of $p \rightarrow \infty f_{g g}$ will define a uniform distribution. The following code defines a 1D Gaussian distribution with mean 10 and standard deviation 2

$i m=1$;

prior $\{$ im $\}$.type='GAUSSIAN' ;

prior $\{\mathrm{im}\} . \mathrm{m} 0=10$;

prior $\{i m\} . s t d=2$;

If not set, the norm is by default set to 2 . The following code defines a $1 \mathrm{D}$ close to uniform distribution in the interval $[8,12]$

$i m=1$;

prior $\{$ im $\}$.type='GAUSSIAN' ; 
prior $\{\mathrm{im}\} . \mathrm{m} 0=10$;

prior $\{i m\} . s t d=2$;

prior $\{$ im $\}$. norm $=60$;

[Figure 1 about here.]

The FFTMA, VISIM and SNESIM type priors all describe a 1D to 3D a priori model defined on a Cartesian grid, which is defined as (for a 3D case)

$i m=1$;

prior $\{$ im $\}$.prior. $\mathrm{x}=[0: 1: 10] ; \% \mathrm{X}$ array

prior $\{$ im\}.prior. $y=[0: 1: 20] ; \%$ Y array

prior $\{$ im\}.prior. $z=[0: 1: 30] ; \% Z$ array

For a $1 \mathrm{D}$ prior only prior $\{\mathrm{im}\}$.prior.x needs to be defined, and for a $2 \mathrm{D}$ prior $\operatorname{prior}\{\mathrm{im}\} \cdot \operatorname{prior} . \mathrm{x}$ and $\operatorname{prior}\{\mathrm{im}\} \cdot \operatorname{prior} . \mathrm{y}$ need to be defined.

Both the FFTMA and VISIM type a priori models describe a multivariate Gaussian a priori pdf, which requires the specification of an a priori mean and covariance model. The a priori mean $\mathrm{m} 0$ can be either a scalar, indicating a constant a priori mean model, or a matrix of the size of the a priori model, allowing for a varying a priori mean model. The model of spatial variability is defined by a, possibly anisotropic, covariance model (equivalent to a semivariogram model) given by the $\mathrm{Cm}$ (or equivalent the Va) field. The specification of the covariance model uses the same notation as used in Pebesma and Wesseling (1998). For example a multivariate Gaussian model defined by 
230

a 2D Spherical type covariance model with sill (or variance) 1, a maximum correlation length of 10 in the direction west to east (i.e. horizontal), and a perpendicular range (i.e. vertical) of 2.5 (hence an anisotropy factor of 0.25 ) and a mean of 10 , is given by

prior $\{\mathrm{im}\} \cdot m 0=10$; prior $\{\mathrm{im}\} . \mathrm{Cm}={ }^{\prime} 1 \mathrm{Sph}(10,90,0.25)^{\prime}$;

FFT Moving Average. prior $\{$ im $\}$. type='FFTMA' defines a spatially correlated multivariate Gaussian a priori model where a priori realizations are generated using the FFT Moving Average generator (FFTMA), Le Ravalec et al. (2000). The FFTMA algorithm is very efficient for generating unconditional realizations from a multivariate Gaussian model. In addition it also allows separation of the random component field and the structural parameters that define spatial correlation. We will discuss the use of this feature in more details later.

A 2D FFTMA type a priori model, on a 200x100 grid, can for example be given by

$i m=1$;

prior $\{$ im $\}$. type $=$ 'FFTMA';

prior $\{$ im\}.prior. $\mathrm{X}=[0: .1: 10] ; \% \mathrm{X}$ array

prior $\{$ im\}.prior. $y=[0: .1: 20] ; \%$ Y array

prior $\{\mathrm{im}\} \cdot \mathrm{m} 0=10$;

prior $\{$ im $\} . V a=' 1 \operatorname{Sph}(10,90, .25)$ ';

Figure 2a shows a set of five realizations from this choice of a priori model. 


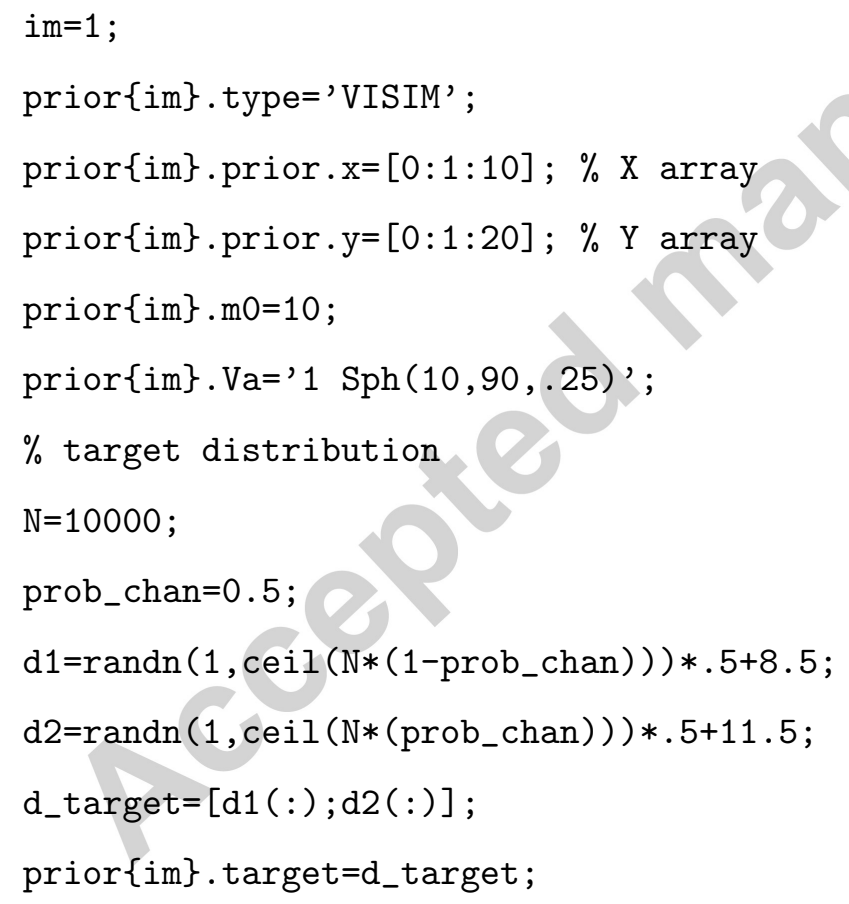
parameters can be given as

VISIM. prior $\{$ im $\}$. type='VISIM' defines a spatially correlated multivariate Gaussian a priori model where a priori realizations are generated using the VISIM algorithm, Hansen and Mosegaard (2008). VISIM can run using sequential Gaussian simulation, in which case the model parameters are assumed normally distributed. It can also run using direct sequential simulation, which allows a (non-Gaussian) target distribution to be set that describes the a priori distribution of the model parameters, while at the same time ensuring that the a priori chosen mean and covariance will be honored.

An a priori model similar to the one described above for the FFTMA type prior, but with an a priori assumption of a bimodal distribution of model

Figure 3 shows a set of five realizations from this VISIM type of a priori model a) without a specification of a target distribution, and b) using a 
target distribution. Once [m,prior]=sippi_prior(prior) has been called once, a data structure will be available as prior $\{\mathrm{im}\} . \mathrm{V}$, which allows access to all options available for running the VISIM algorithm. See Hansen and Mosegaard (2008) for more details on VISIM.

[Figure 3 about here.]

The FFTMA and VISIM type prior models only allow reproducing the first two moments of the distribution describing the spatial variability, the mean and the covariance (i.e. Gaussian variability between sets of two data points). Maximum entropy is implicitly assumed in higher order moments, Journel and Zhang (2006). This is the reason why geological structures such as for example meandering channels cannot be reproduced by Gaussian statistics. To achieve this one can make use of statistical models based on higher order moments.

SNESIM. prior $\{$ im $\}$.type='SNESIM' defines an a priori model based on a higher order statsistical moments (a multiple point statistical model) describing spatial variability as inferred from a training image.

There are several methods that allow sampling from an a priori model defined by multiple point statistics. Here, we use the SNESIM algorithm, originally developed by Strebelle $(2000,2002)$, and we make use of the implementation available in the SGeMS software package, Remy et al. (2008). It works by initially extracting a multiple point based statistical model from a training image. Then sequential simulation is used to generate realizations of this statistical model. 
Optionally the scaling and rotation field can be speified. prior $\{$ im $\}$. scaling $=2$ scales the axis of the training image such that spatial structures appears twice as large. prior $\{\mathrm{im}\}$.rotation $=45$ rotates the training image 45 degrees clockwise.

A 2D SNESIM type prior with the training image 'channels.ti' (Figure 4) rotated 30 degrees and scaled by a factor of 0.75 , with two categories ('0' and ' 1 '), and where the first category ' 0 ' reflect a model parameter value of 8 , and the second category ' 1 ' reflect a value of 12 , is given by

$i m=1$;

prior $\{$ im $\}$.type='SNESIM';

prior $\{\operatorname{im}\} . x=[0: .1: 10]$;

prior $\{\operatorname{im}\} \cdot y=[0: .1: 20]$;

prior $\{$ im\}.ti=' channels.ti';

prior $\{$ im $\}$. index_values $=\left[\begin{array}{ll}0 & 1\end{array}\right] ; \%$ optional

prior $\{$ im $\} . m_{-}$values $=\left[\begin{array}{ll}8 & 12\end{array}\right] ; \%$ optional

prior $\{$ im $\}$.scaling $=.75 ; \%$ optional

prior $\{$ im\}.rotation $=30 ; \%$ optional

Figure 5 shows a set of five realizations from this choice of a priori model. Once $[\mathrm{m}$, prior] =sippi prior (prior) has been called, a data structure will be available as prior $\{\mathrm{im}\}$.S which allow access to all options available for running the SNESIM algorithm as implemented in SGeMS. See Remy et al. (2008) for more details on setting up the SNESIM algorithm.

[Figure 4 about here.]

[Figure 5 about here.] 
Distribution transform. A normal score transform can be defined for any of the Gaussian based a priori models, that allow the transformation of the normally distributed model parameters to any desired distribution, see e.g. Goovaerts (1997). It requires only that the user defines the 'target' distribution, in form of a sample of the target distribution in the d_target field. For example a bimodal distribution with increased probability of values around 8.5 and 11.5 , can be given by

$\mathrm{N}=10000$;

prob_chan $=0.5$;

$\mathrm{d} 1=\operatorname{randn}\left(1, \operatorname{ceil}\left(\mathrm{N} *\left(1-\right.\right.\right.$ prob_chan $\left.\left._{)}\right)\right) * .5+8.5$;

$\mathrm{d} 2=\operatorname{randn}(1, \operatorname{ceil}(\mathrm{N} *($ prob_chan $))) * .5+11.5$;

d_target $=[\mathrm{d} 1(:) ; \mathrm{d} 2(:)]$;

prior $\{$ im $\} . d_{-}$target=d_target;

Note that the number $N$ here reflects the size of the sample generated and used to describe the target distribution in the d_target field, and can be chosen arbitrarily large. The larger the sample, the better accuracy of reflecting a specific distribution. An example of combining this distribution transform with the FFTMA type prior used to generate Figure $2 \mathrm{a}$ is shown in Figure 2b.

Note that when using the VISIM type prior one can use a target distribution directly, while ensuring that the chosen a priori covariance model is still honored. Using the distribution transform with the FFTMA prior will not preserve the properties of the a priori chosen covariance model.

Randomizing the model of spatial variability. As mentioned for the 'FFTMA' prior type model, the structural parameters that describe the a priori model 


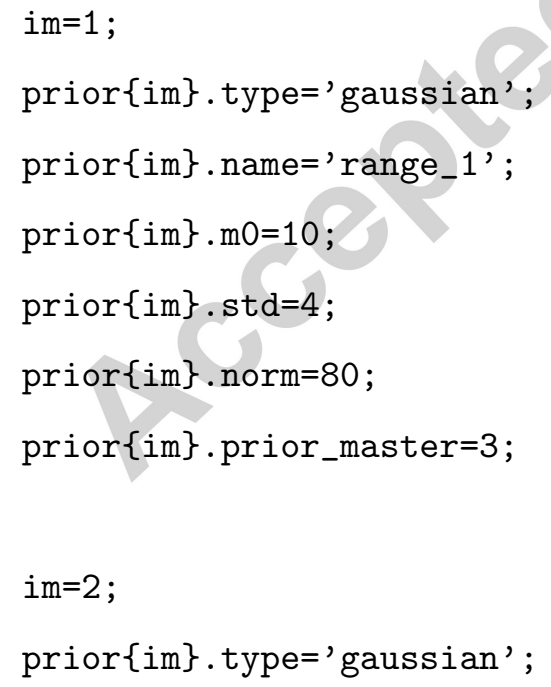




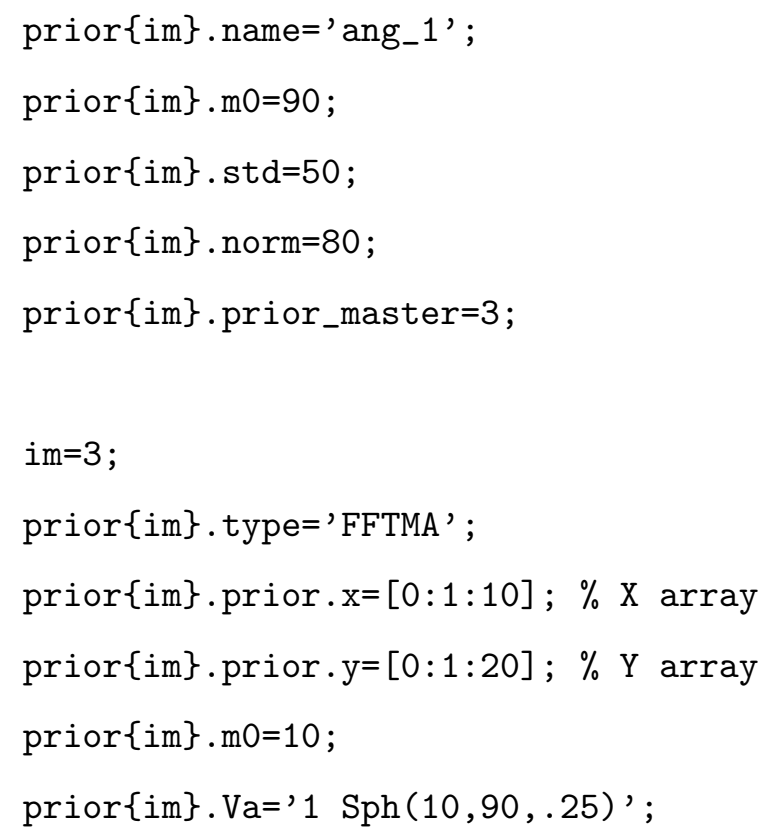

Figure 2c shows an example of 5 realizations from such an a priori model.

\subsubsection{A random walk in the a priori model space}

To perform a random walk in the prior probability space, as needed by the extended Metropolis sampler, we make use of sequential Gibbs sampling, Hansen et al. (2012). An application of the sequential Gibbs sampler essentially amounts to selecting a subset, which can be any subset of model parameters, and simulate these conditional to the rest of the model parameters. The number of chosen model parameters in the subset controls the exploratory nature (i.e. step-length) of the sequential Gibbs sampler (which controls the degree of correlation between successive realizations), and hence the efficiency of the extended Metropolis sampler. All properties of the sequential Gibbs sampler is controlled by seq-gibbs structure, which is a field in the prior data structure. Two different methods for selecting the subset 
of model parameters for conditional re-simulation have been implemented.

Box type subset. If prior $\{\mathrm{im}\}$. seq_gibbs . type=1, then a line/rectangle/cube of model parameters (for the 1D, 2D and 3D case respectively) is selected as the subset used for conditional re-simulation. The width of the box is defined by prior $\{$ im $\}$.seq_gibbs.step. For example a box with dimension $2 \times 3 \times 4$ (in the units of the prior model considered - typically meters) is given by prior $\{i m\}$.seq_gibbs.step $=\left[\begin{array}{lll}2 & 3 & 4\end{array}\right]$. The center of the 'box' is chosen randomly

Randomly selected subset. If prior $\{\mathrm{im}\}$. seq_gibbs . type $=2$, then a randomly selected number of the total number of model parameters is selected as the subset used for conditional resimulation. The number of data used for conditional re-simulation is given by $\operatorname{prior}\{i m\}$. seq_gibbs.step. If prior $\{\mathrm{im}\}$.seq_gibbs.step is smaller than 1, it is interpreted as a percentage of the total number of model parameters.

As an example, five iterations of sequential Gibbs sampling can in SIPPI be performed using iterative calls to sippi_prior as

[m_current,prior] =sippi_prior(prior);

for $i=1: 5$

[m_proposed, prior] =sippi_prior (prior,m_current); end

Figures 6 and Figure 7 shows examples of using sequential Gibbs sampling with a box type selection and random type selection of model parameters for conditional re-simulation, respectively. The a priori model is in both cases 
the same as the one used to generate the unconditional realizations of Figure

3. The options for the box type re-simulation is

prior $\{i m\}$.seq_gibbs.type $=1$;

prior $\{$ im $\}$.seq_gibbs. step $=\left[\begin{array}{ll}4 & 4\end{array}\right]$;

while the options for the random type re-simulation, with only $0.5 \%$ of the total number of model parameter used as conditional data for re-simulation, is

prior $\{i m\}$. seq_gibbs . type $=2$;

prior $\{$ im $\}$.seq_gibbs.step $=0.995$;

The sequential Gibbs sampler can be used with the FFTMA, VISIM, and SNESIM types a priori models. For the 1D GAUSSIAN type a priori model we use an alternate method. Given a current realization of the a priori model, a step length between 0 and 1 will generate a new realization of the prior, in the vicinity if the current realization. A step length of '0' indicates no change, while a step length of ' 1 ' will generate a new unconditional realization of the a priori model.

Figure 8 shows the first 300 iterations when sampling the same a priori model as sampled in Figure 1 using a step length of 0.25 , prior $\{$ im $\}$. seq_gibbs . step=0 25 .

After 100000 iterations the histogram of the sampled model parameters resemble that of Figure 1, and is therefore not shown here.

[Figure 6 about here.]

[Figure 7 about here.]

[Figure 8 about here.] 
3.2. Data, data uncertainties, modelization errors and the likelihood function

Observed data must be given in the data data structure along with a description of the noise model. As for the prior structure, the data structure may consist of many types of data, where each data type number id is defined in the data $\{i d\}$ structure. Observed data are stored in the d_obs field. Uncorrelated uncertainty can be given either in the form of standard deviation, d_std, or variance, d_var. A simple data structure with such uncorrelated uncertainties can be given by

id=1;

data $\{i d\} . d \_o b s=\left[\begin{array}{lll}0 & 3 & 4\end{array}\right]^{\prime}$;

data $\{i d\} . d \_s t d=\left[\begin{array}{lll}2 & 2 & 2\end{array}\right]^{\prime}$;

If the data uncertainties are uncorrelated, the noise model can be described by a generalized Gaussian model as defined in Eq. 7, if the norm of the generalized Gaussian is set by data $\{$ id $\}$.norm. If not specified a Gaussian noise model (using a norm of 2) is chosen by default.

The noise model can also be given in form of a correlated Gaussian model, for both the data noise, $\mathbf{C}_{\mathrm{d}}$ and the modelization error, $\mathbf{C}_{T}$. The following will for example specify a correlated Gaussian noise model:

$i d=1$

data $\{i d\} \cdot d_{-} o b s=\left[\begin{array}{lll}0 & 3 & 4\end{array}\right]^{\prime}$;

data $\{$ id $\} . C d=\left[\begin{array}{lllllllllll}4 & 0 & .1 & ; & 0 & 4 & 0 & ; & .1 & 0 & 4\end{array}\right]$;

If a Gaussian model for the modelization error, $\mathcal{N}\left(\mathbf{d}_{T}, \mathbf{C}_{T}\right)$, is available it can be specified as 
data $\{i d\} . d t=\left[\begin{array}{lll}0 & -1 & 0\end{array}\right]^{\prime} ;$

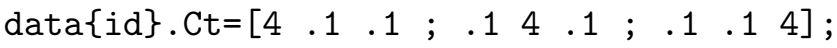

where $\mathbf{d}_{T}$ is a bias correction.

One can choose to consider only a subset of the available data using the i_use field. To use for example only data number 1 and 3 use

$i d=1$;

$\operatorname{data}\{i d\} . d \_o b s=\left[\begin{array}{lll}0 & 3 & 4\end{array}\right]^{\prime}$;

$\operatorname{data}\{i d\}$. i_use $=\left[\begin{array}{ll}1 & 3\end{array}\right]$;

Once the data structure has been setup in data, the log-likelihood and the likelihood of a given data response $d$ can be computed using

$[\log L, L$, data $]=$ sippi_likelihood (d, data);

\subsection{The forward problem}

The forward problem is naturally problem dependent, and to use SIPPI, the user needs to supply the solution to the forward problem, wrapped in the m-file sippi_forward.m.

The input to sippi_forward.m is the forward, data and prior Matlab structures. The forward structure can contain information on how to solve the forward problem. The output must be the data obtained by solving the forward problem, in form of the data structure d which must be of the same length as the data structure, and each entry of $d\{i d\}$ must have the same size as data $\{$ id $\} . d \_o b s$, or the size of data $\{i d\}$. i_use if a data subset is specified.

As an alternative for providing sippi_forward, one can provide a generic name for the m-file solving the forward problem by setting forward.forward_function. 
sippi_rejection(data, prior, forward, options) ; 
By default the $L_{\max }=1$, see Eq. 6 . This can be manually changed by providing the options.mcmc.Lmax.

\subsubsection{Metropolis sampling}

All available a priori model types and noise models in SIPPI work seamlessly as part of the extended Metropolis algorithm. The extended Metropolis sampling algorithm can be applied using

options=sippi_metropolis (data,prior, forward, options);

The options structure define some properties of how the Metropolis algorithm will run.

options.mcmc.nite determines the number of iterations of the extended Metropolis algorithm. options.mcmc.i_sample sets how often the current model is saved to disc, measured in number of iterations. options.mcmc.i_plot sets number of iterations between updating figures showing the progress of the algorithm. If any of these parameters are not set, the following values will be chosen by default

options.mcmc.nite $=30000$;

options.mcmc.i_sample= 500;

options.mcmc.i_plot: 50

Perturbation strategy. The choice of the number of model parameters to be perturbed in each iteration of the extended Metropolis algorithm can have large impact on its computational performance. By default a random type of model parameter is perturbed in each iteration. Thus if 3 types of a priori models have been specified in prior $\{1\}$, prior $\{2\}$, and prior $\{3\}$, the probability of perturbing each individual type of prior model in each iteration is 
$1 / 3$. This default behaviour can be changed by choosing a perturbation strategy. options.mcmc.pert_strategy.i_pert selects the number of a prior model types to perturb, and options.mcmc.pert_strategy.i_pert_freq set the relative frequency of each selected type of prior model. Thus, to perturb prior model 1 and 3 (but never model 2), such that prior model 3 is perturbed 9 times as often as prior type 1, one could use

options.mcmc.pert_strategy.i_pert=[1 3 [ ;

options.mcmc.pert_strategy.i_pert_freq=[1 9];

Automatic adjustment of the exploration rate (step length). The exploratory nature of the Metropolis sampling algorithm, controlled by the 'step length', has large impact on its computational demands. A small step-length provides a dense local sampling, but the algorithm will use many iterations to move away from the initial point, i.e. a less exploratory algorithm. A large step length will lead to a very exploratory sampling algorithm that will not get trapped in local minima, but many models that are proposed will be rejected. Gelman et al. (1996) argues that a step-length leading to an acceptance rate in the Metropolis sampler of about 20-40\% will lead to a good compromise between exploration and rejection rate. SIPPI allows automatic detection of the step length leading to an acceptance rate specified by prior $\{i m\}$.seq-gibbs.P_target, using the method given by Cordua et al. (2012). Note that the Metropolis sampler will not sample the a posteriori pdf correct until the step-length is fixed, and unchanged. Therefore one can set the number of initial iterations in which adjustment of the step length is allowed using prior $\{\mathrm{im}\}$.seq_gibbs.i_update_step_max. After this, actual sampling of the a posteriori pdf will start, if the algorithm has reached 
burn-in. prior $\{i m\}$.seq_gibbs.i_update_step sets the number of iterations between updating the step length. prior $\{$ im $\}$.seq_gibbs.step_min and prior $\{i m\}$.seq_gibbs.step_max determine the minimum and maximum allowed step length.

The default choice of the step length is to use infinitely long step-length, resulting in a prior sampler generating statistically independent realization of the prior in each iteration.

As an example, a preferred acceptance ratio of 0.3 , adjusted in the first 1000 iterations, allowing step lengths in the interval 1 to 100 (using type 1 data subset), can be specified using:

prior $\{i m\}$.seq_gibbs . type $=1$;

prior $\{i m\}$.seq_gibbs.step_min=1;

prior $\{$ im\}.seq_gibbs.step_max $=100$;

prior $\{$ im $\}$.seq_gibbs.step $=100$;

prior $\{i m\}$.seq_gibbs.i_update_step_max $=1000$;

prior $\{$ im $\}$.seq_gibbs.P_target $=0.3$;

\subsubsection{Linear Gaussian inverse Problems}

In the specific case where the forward problem is linear, and the a priori model Gaussian, as defined by the VISIM of FFTMA type a priori model, the a posteriori pdf can be sampled directly without the need for the Metropolis algorithm using

[m_reals,m_est, Cm_est $]=$ sippi_least_squares (data,prior,forward,n_reals, lsq_type);

$\mathrm{n} \_$reals sets how many a posteriori realizations, as output in $\mathrm{m} \_r e a l s$, that are generated. 1sq-type determines the method used to solve sample the a 
posteriori pdf. m_est and $\mathrm{Cm}_{-}$est are the a posteriori mean and covariance as given by Eq. 5, and are only available if least squares types of inversion is performed.

Three methods described previously, are available to generate samples of the a posteriori pdf, and can be selected by setting the the 1sq_type argument when calling sippi_least_squares.

lsq_type=' 'lsq' use classical least-squares inversion where the complete Gaussian a posteriori pdf can be analytically derived in form of a posteriori mean and covariance of Eqs. 4-5. Then Cholesky decomposition of the a posterior covariance is used to generated realizations of the a posteriori pdf. Isq_type=' error_sim' make use of kriging simulation through error simulation to generate a sample of the a posteriori pdf, Journel and Huijbregts (1978); Gloaguen et al. (2005a,b); Hansen and Mosegaard (2008).

Isq_type='visim' make use of the VISIM algorithm for sampling the a posteriori pdf, Hansen and Mosegaard (2008). The type of prior model must be chosen as a VISIM type prior model. If the target distribution is set as prior $\{\mathrm{im}\}$.target then VISIM runs as a direct sequential simulation algorithm. If it is not set, VISIM will run as a sequential Gaussian simulation algorithm.

\section{Conclusions}

A generic Matlab and Gnu Octave toolbox for sampling the a posteriori pdf of linear and non-linear inverse problems has been presented. Prior information about the model parameters can be described by any number of the following types of a priori models: 1) 1D arbitrarily distributed pdf, 2) 
1D-3D multivariate Gaussian pdf as sampled using the FFTMA method, 3) 1D-3D multivariate Gaussian model as sampled using the VISIM algorithm (utilizing both sequential Gaussian simulation and direct sequential simulation), or 4) 1D-3D multiple-point based statistical models as sampled using the SNESIM algorithm.

For linear Gaussian inverse problems the a posteriori pdf can be sampled using 1) traditional least squares inversion combined with Cholesky decomposition of the a posteriori covariance, 2) sequential Gaussian simulation, 3) direct sequential simulation and 4) Gaussian simulation through error simulation.

For non-linear and non-Gaussian inverse problems the a posteriori pdf can be sampled using the rejection sampler or the extended Metropolis sampler. The computational efficiency of the extended Metropolis sampler can be controlled by using a flexible perturbation mechanism, based on sequential Gibbs sampling, allowing arbitrary long or short step length. The choice of the step length can optionally be automatized.

The combination of the FFTMA method with the extended Metropolis algorithm allows treating the properties describing the Gaussian a priori model, to be treated as model parameters, and thus inferred as part of the inversion.

\section{Acknowledgement}

We thank DONG for financial support. Interfacing geostatistical algorithms has been done using the mGstat toolbox, http://mgstat.sourceforge.net/. SIPPI source code can be downloaded from http://sippi.sourceforge.net/. 
Cordua, K. S., Hansen, T. M., Mosegaard, K., 2012. Monte Carlo full waveform inversion of crosshole GPR data using multiple-point geostatistical a priori information. Geophysics 77, H19-H31.

Gelman, A., Roberts, G., Gilks, W., 1996. Efficient metropolis jumping rules. In: Bernardo, J., Berger, K., Dawid, A., Smith, A. (Eds.), Bayesian Statistics 5,. Clarendon press, Oxford., pp. 599-608.

Gloaguen, E., Marcotte, D., Chouteau, M., 2005a. A non-linear tomographic inversion algorithm based on iterated cokrigriging and conditional simulations. In: Leuangthong, O., Deutsch, C. (Eds.), Geostatistics Banff 2004. Vol. 1. Springer, pp. 409-418.

Gloaguen, E., Marcotte, D., Chouteau, M., Perroud, H., 2005b. Borehole radar velocity inversion using cokriging and cosimulation. Journal of Applied Geophysics 57 (4), 242-259.

Gómez-Hernández, J., Froidevaux, R., Biver, P., 2005. Exact conditioning to linear constraints in kriging and simulation. In: Leuangthong, O., Deutsch, C. (Eds.), Geostatistics Banff 2004. Vol. 2. Springer, pp. 999-1005.

Goovaerts, P., 1997. Geostatistics for natural resources evalutaion. Applied Geostatistics Series. Oxford University Press.

Hansen, T., Cordua, K., Looms, M., Mosegaard, K., 201x. SIPPI : A Matlab toolbox for Sampling the solution to Inverse Problems with complex Prior Information: Part 2, Application to cross hole GPR tomography. Computers \& Geosciences. 
Hansen, T. M., Cordua, K. C., Mosegaard, K., 2012. Inverse problems with non-trivial priors - efficient solution through sequential Gibbs sampling. Computational Geosciences 16 (3), 593-611.

Hansen, T. M., Journel, A. G., Tarantola, A., Mosegaard, K., 2006. Linear inverse Gaussian theory and geostatistics. Geophysics 71 (6), R101-R111.

Hansen, T. M., Mosegaard, K., 2008. VISIM: Sequential simulation for linear inverse problems. Computers and Geosciences 34 (1), 53-76.

Hansen, T. M., Mosegaard, K., Cordua, K. C., 2008. Using geostatistics to describe complex a priori information for inverse problems. In: Ortiz, J. M., Emery, X. (Eds.), VIII International Geostatistics Congress. Vol. 1. Mining Engineering Department, University of Chile, pp. 329-338.

Hastings, W., 1970. Monte Carlo sampling methods using Markov chains and their applications. Biometrika 57 (1), 97.

Irving, J., Singha, K., 2010. Stochastic inversion of tracer test and electrical geophysical data to estimate hydraulic conductivities. Water Resour. Res 46.

Journel, A., Zhang, T., 2006. The Necessity of a Multiple-Point Prior Model. Mathematical Geology 38 (5), 591-610.

Journel, A. G., 1999. Conditioning geostatistical operations to nonlinear volume averages,. Mathematical Geology 31, 931-953.

Journel, A. G., Huijbregts, C. J., 1978. Mining Geostatistics. Academic Press. 
Le Ravalec, M., Noetinger, B., Hu, L. Y., 2000. The FFT moving average (FFT-MA) generator: An efficient numerical method for generating and conditioning Gaussian simulations. Mathematical Geology 32 (6), 701-723.

Mariethoz, G., Renard, P., Caers, J., 2010. Bayesian inverse problem and optimization with iterative spatial resampling. Water Resources Research 46 (11), W11530.

Metropolis, N., Rosenbluth, M., Rosenbluth, A., Teller, A., Teller, E., 1953. Equation of state calculations by fast computing machines. J. Chem. Phys. 21, 1087-1092.

Mosegaard, K., Sambridge, M., 2002. Monte Carlo analysis of inverse problems. Inverse Problems 18 (3), 29-54.

Mosegaard, K., Tarantola, A., 1995. Monte Carlo sampling of solutions to inverse problems. Journal of Geophysical Research 100 (B7), 12431-12447.

Mosegaard, K., Tarantola, A., 2002. Probabilistic approach to inverse problems. In: Lee, W., Kanamori, H., Jennings, P., Kisslinger, C. (Eds.), International handbook of earthquake and engineering seismology. Vol. 81A. WHK Lee et al, Ch. 16, pp. 237-265.

Pebesma, E. J., Wesseling, C. G., 1998. Gstat: a program for geostatistical modelling, prediction and simulation. Computers \& Geosciences 24 (1), $17-31$.

Remy, N., Boucher, A., Wu, J., 2008. Applied Geostatistics with SGeMS: A User's Guide. Cambridge University Press. 
608

609

610

611

612

613

614

615

616

617

618

Strebelle, S., 2000. Sequential simulation drawing structures from training images. Ph.D. thesis, Stanford University.

Strebelle, S., 2002. Conditional simulation of complex geological structures using multiple-point statistics. Math. Geol 34 (1), 1-20.

Tarantola, A., 2005. Inverse Problem Theory and Methods for Model Parameter Estimation. SIAM.

Tarantola, A., Valette, B., 1982a. Generalized nonlinear inverse problems solved using the least squares criterion. Rev. Geophys. Space Phys 20 (2), 219-232.

Tarantola, A., Valette, B., 1982b. Inverse problems= quest for information. J. geophys 50 (3), 150-170. 


\section{List of Figures}

1 Histogram of 100000 unconditional realizations from a generalized Gaussian, GAUSSIAN type prior model with norm 60 and

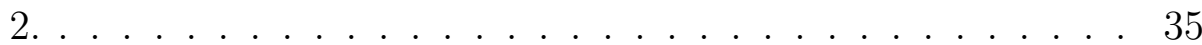

2 Unconditional realizations from a FFTMA type priori model with a) Gaussian distribution, b) target distribution, and c) random structural parameters (range and rotation). . . . . . . 36

3 Unconditional realizations from a VISIM type a priori model with with a) Gaussian distribution, b) target distribution. . . 37

4 Example of a training image for use with the SNESIM type a priori model. . . . . . . . . . . . . . . . . . . 38

5 Unconditional realizations from a SNESIM type a priori model. 39

6 top) Random walk using sequential Gibbs sampling with box type re-simulation, and the VISIM type a priori model. bottom) Black pixels indicate the model parameters that are simulated conditional to the value of the model parameters indicated by pixels. . . . . . . . . . . . . . . . . . 40

7 top) Random walk using sequential Gibbs simulation with random choice of model parameters for resimulation, and the VISIM type a priori model. bottom) Black pixels indicate the model parameters that are simulated conditional to the value of the model parameters indicated by white pixels. . . . . . . . 41

8 The first 300 realizations from the GAUSSIAN type a priori model with a mean of 10 , and a norm 60 and 2 respectively, using a step length of $0.25 \ldots$. . . . . . . . . . . . . . . 42 


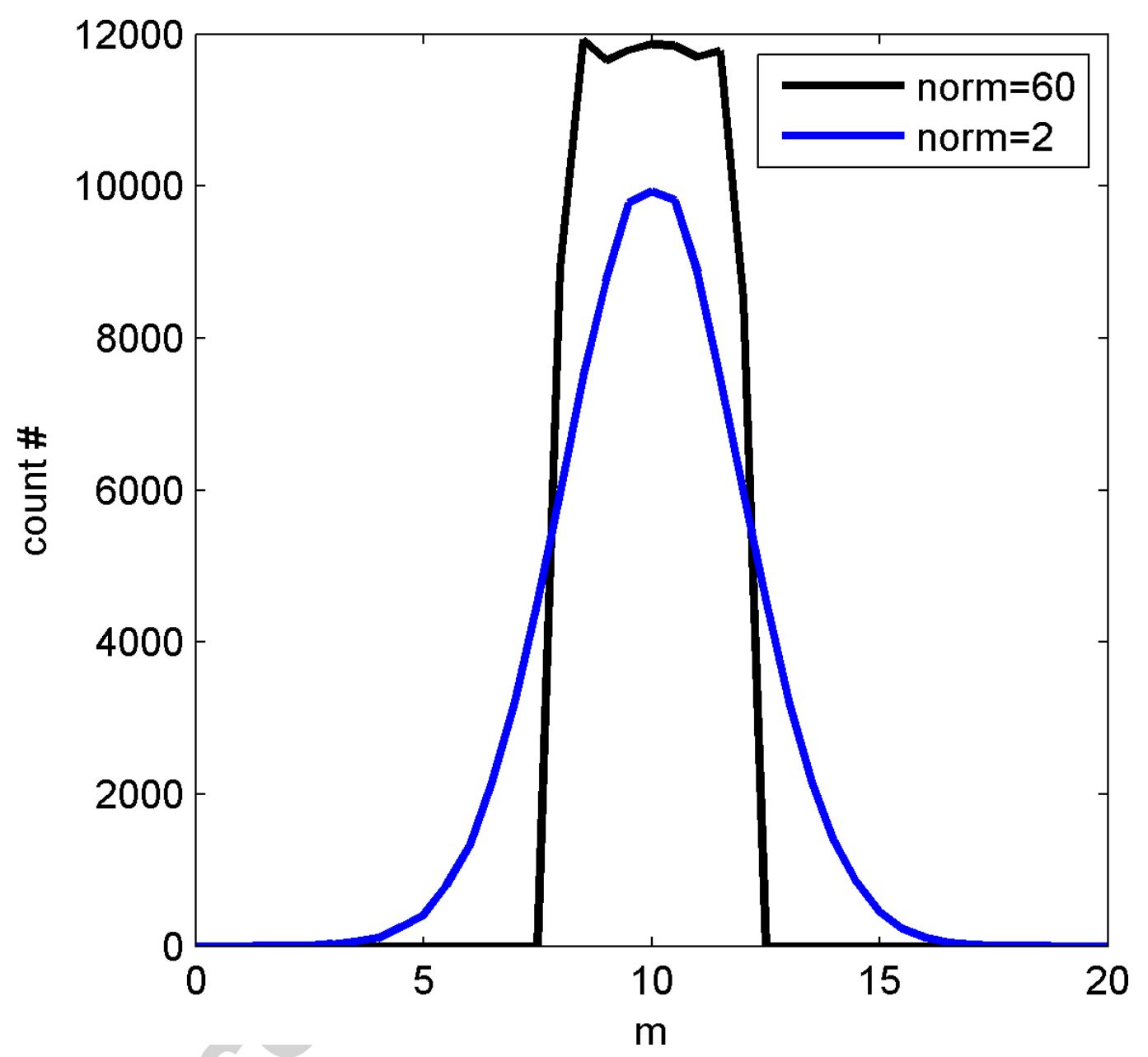

Figure 1: Histogram of 100000 unconditional realizations from a generalized Gaussian, GAUSSIAN type prior model with norm 60 and 2 . 
a)
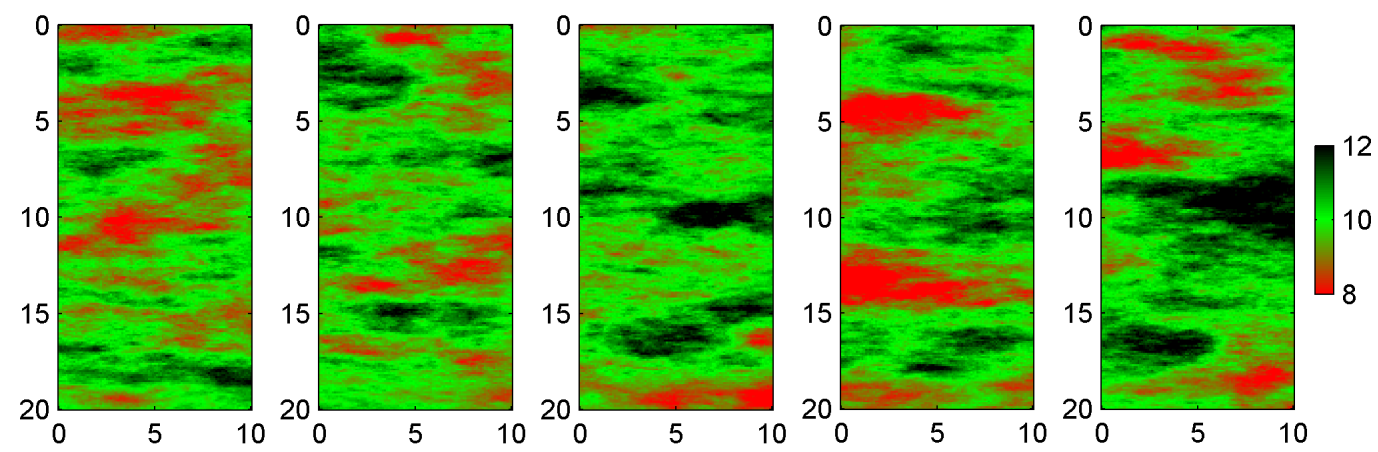

b)
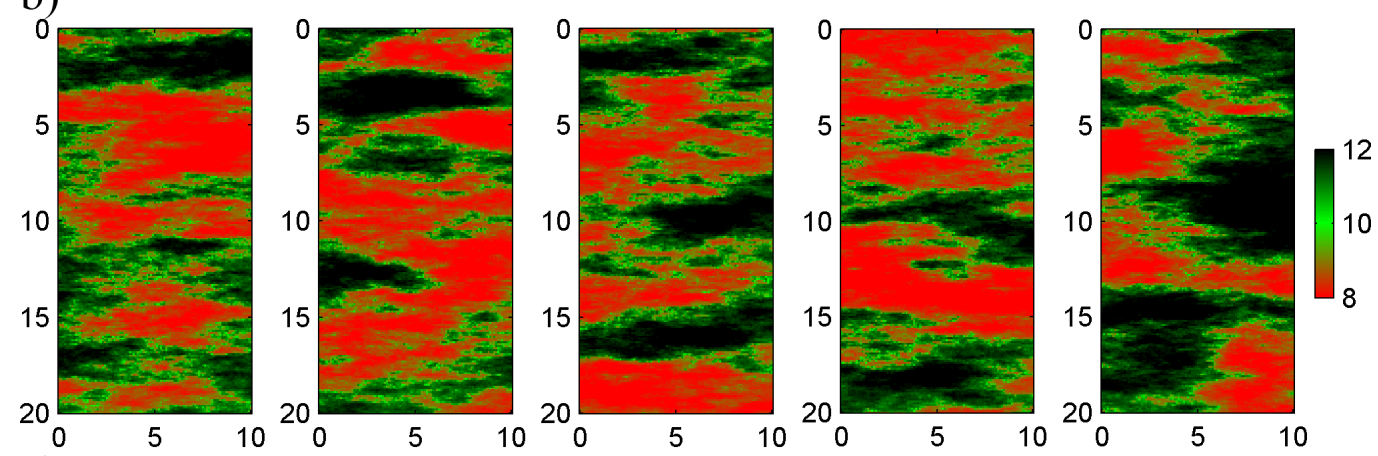

C)
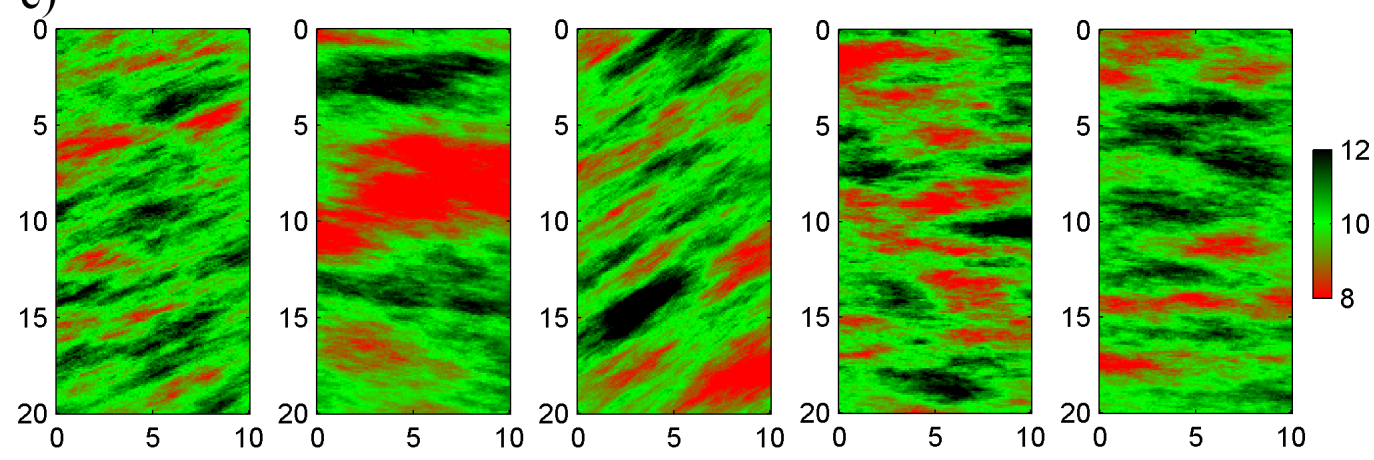

Figure 2: Unconditional realizations from a FFTMA type priori model with a) Gaussian distribution, b) target distribution, and c) random structural parameters (range and rotation). 
a)
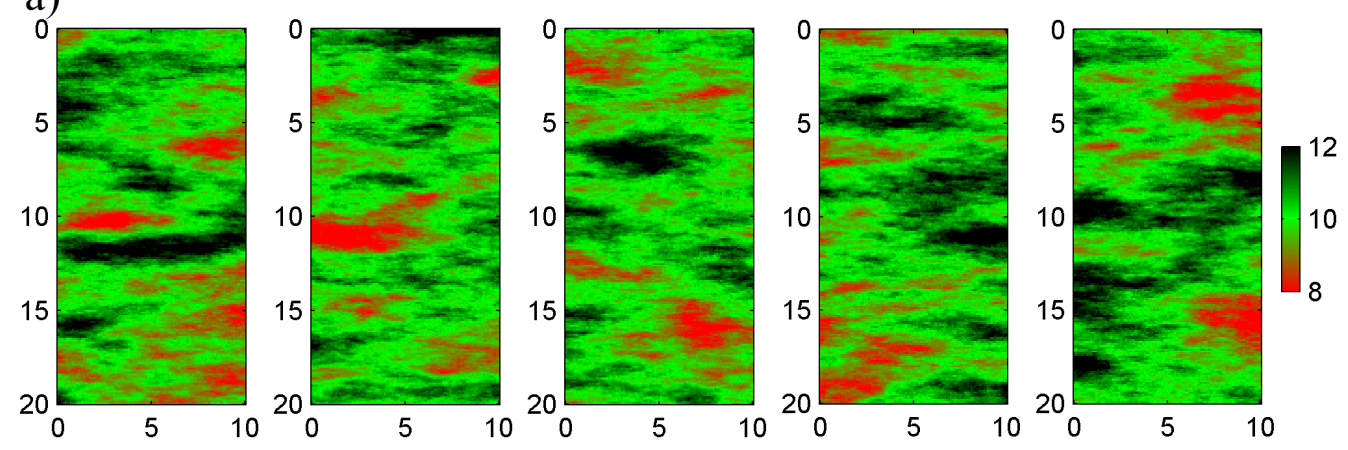

b)
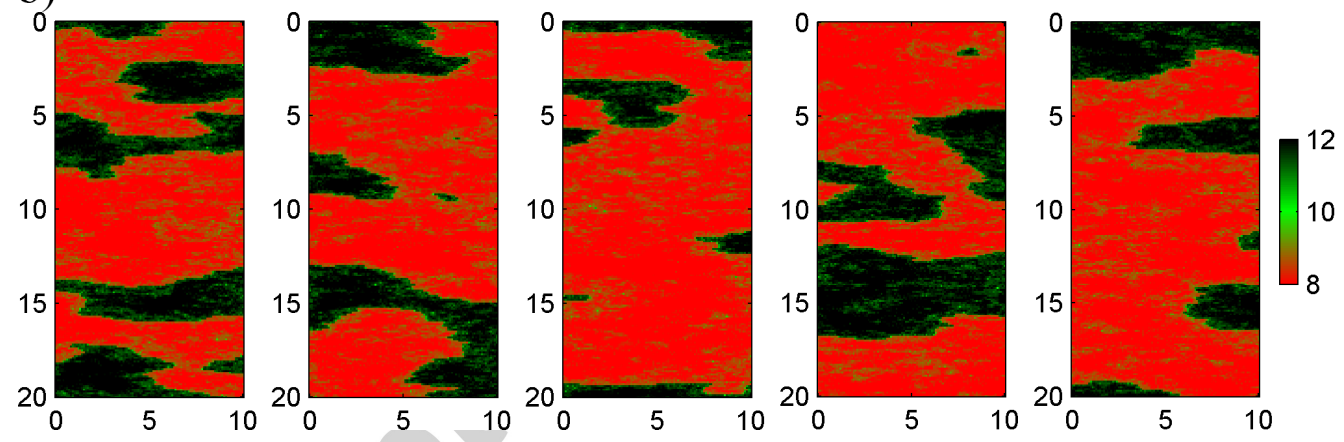

Figure 3: Unconditional realizations from a VISIM type a priori model with with a) Gaussian distribution, b) target distribution. 


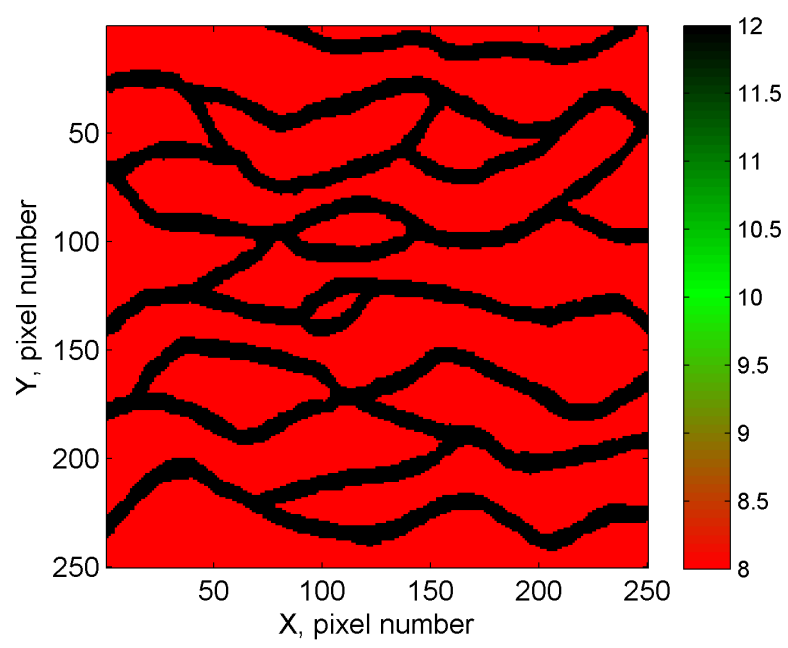

Figure 4: Example of a training image for use with the SNESIM type a priori model. 

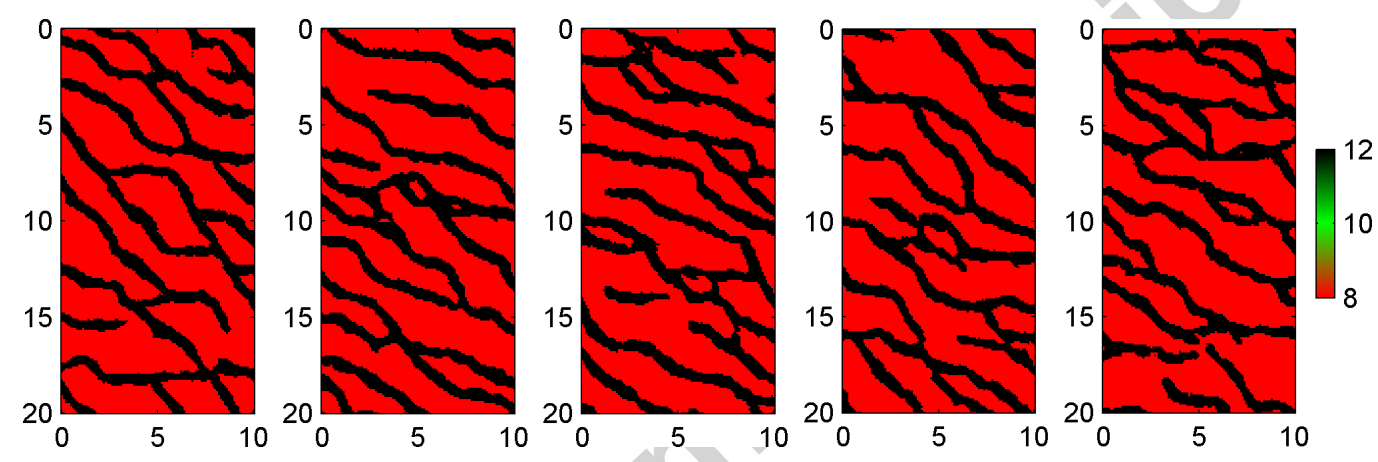

Figure 5: Unconditional realizations from a SNESIM type a priori model. 

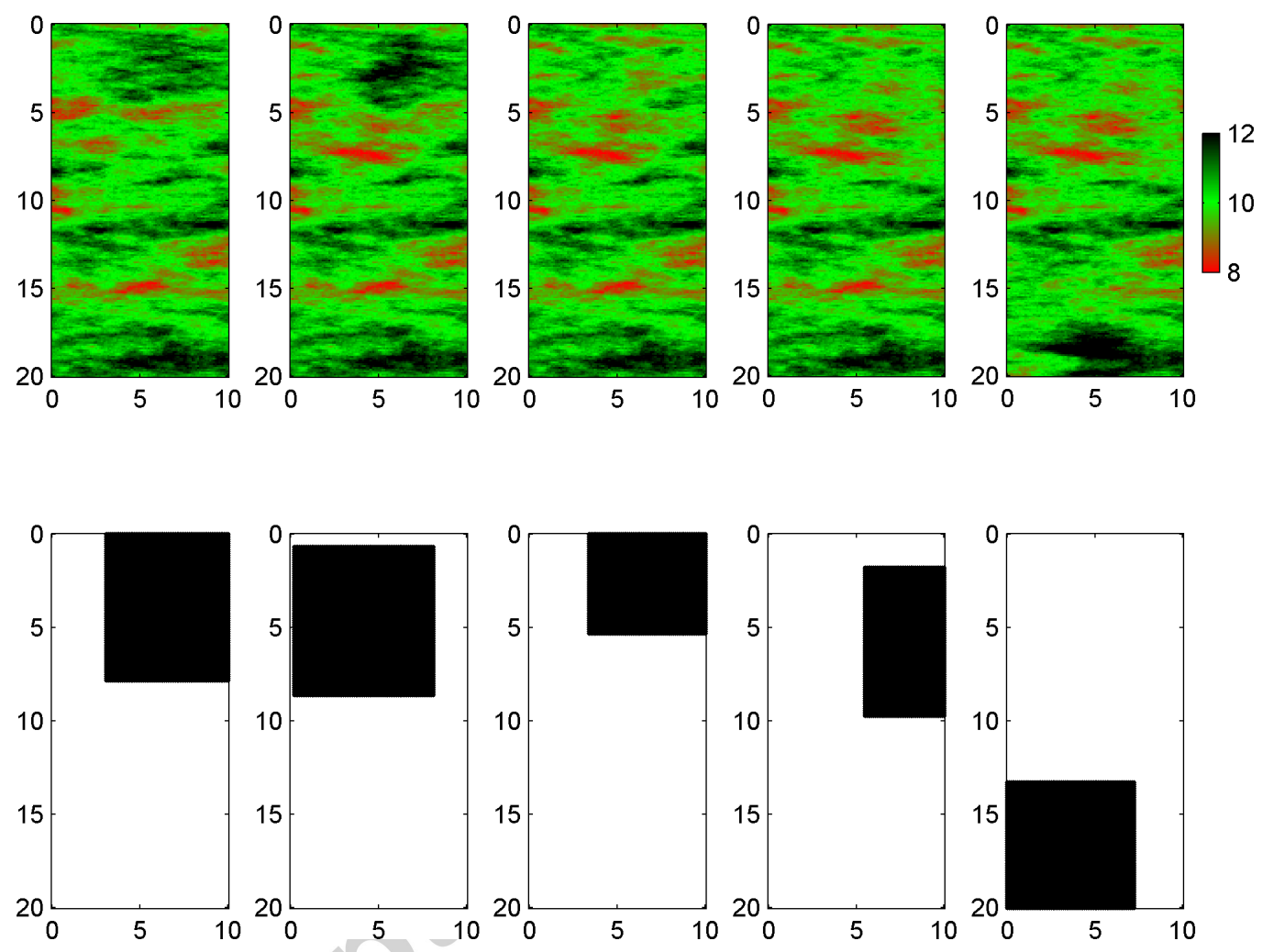

Figure 6: top) Random walk using sequential Gibbs sampling with box type re-simulation, and the VISIM type a priori model. bottom) Black pixels indicate the model parameters that are simulated conditional to the value of the model parameters indicated by pixels. 

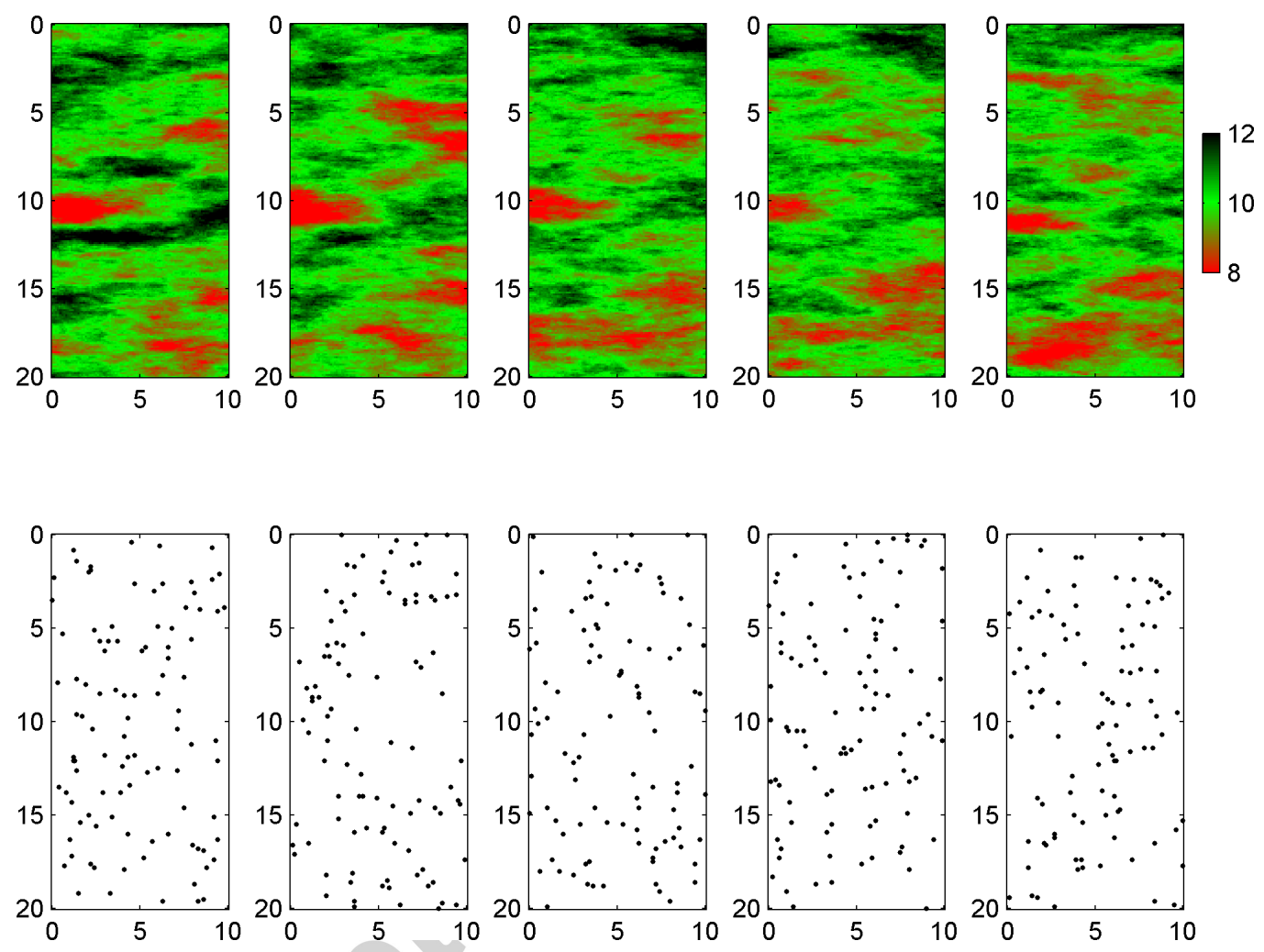

Figure 7: top) Random walk using sequential Gibbs simulation with random choice of model parameters for resimulation, and the VISIM type a priori model. bottom) Black pixels indicate the model parameters that are simulated conditional to the value of the model parameters indicated by white pixels. 


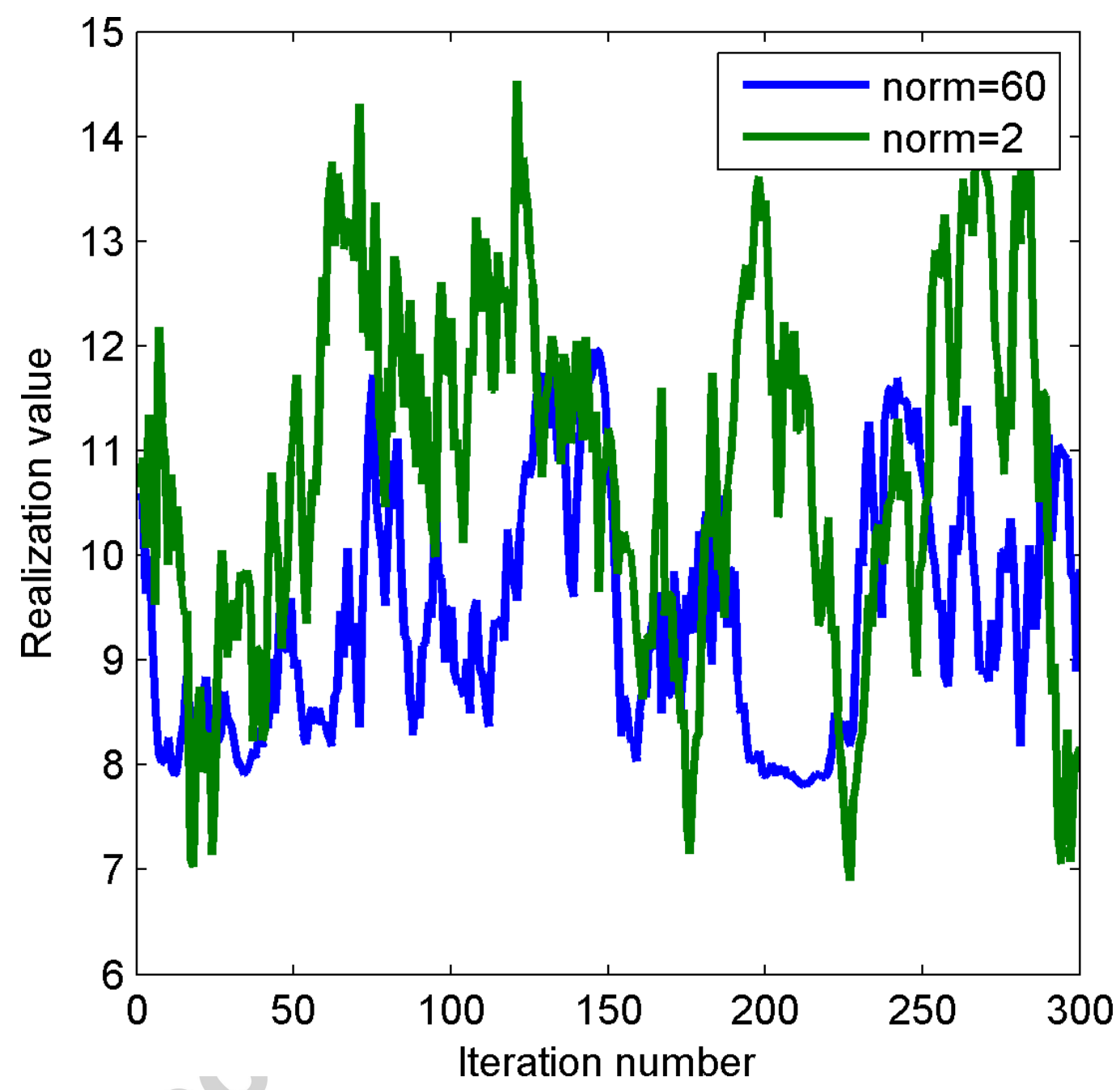

Figure 8: The first 300 realizations from the GAUSSIAN type a priori model with a mean of 10, and a norm 60 and 2 respectively, using a step length of 0.25 . 